\title{
A general method for implementing vibrationally adiabatic mixed quantum-classical simulations
}

\author{
Ward H. Thompson ${ }^{\text {a) }}$ \\ Department of Chemistry, University of Kansas, Lawrence, Kansas 66045
}

(Received 19 June 2002; accepted 22 October 2002)

\begin{abstract}
An approach for carrying out vibrationally adiabatic mixed quantum-classical molecular dynamics simulations is presented. An appropriate integration scheme is described for the vibrationally adiabatic equations of motion of a diatomic solute in a monatomic solvent and an approach for calculating the adiabatic energy levels is presented. Specifically, an iterative Lanczos algorithm with full reorthogonalization is used to solve for the lowest few vibrational eigenvalues and eigenfunctions. The eigenfunctions at one time step in a mixed quantum-classical trajectory are used to initiate the Lanczos calculation at the next time step. The basis set size is reduced by using a potential-optimized discrete variable representation. As a demonstration the problem of a homonuclear diatomic molecule in a rare gas fluid $\left(\mathrm{N}_{2}\right.$ in $\left.\mathrm{Ar}\right)$ has been treated. The approach is shown to be efficient and accurate. An important advantage of this approach is that it can be straightforwardly applied to polyatomic solutes that have multiple vibrational degrees-of-freedom that must be quantized. (c) 2003 American Institute of Physics. [DOI: 10.1063/1.1528891]
\end{abstract}

\section{INTRODUCTION}

Quantum-mechanical effects associated with nuclei are important in a variety of condensed phase systems including proton and hydrogen atom transfer, vibrational relaxation and dephasing, and spectroscopy. However, except for a few special cases, a fully quantum-mechanical treatment of systems involving more than a handful of atoms is not feasible. Fortunately, in many cases the relevant quantum effects are associated with one or only a few atoms. This has motivated the development of mixed quantum-classical (QC) and semiclassical methods that can account for the relevant quantum effects in such cases, even in systems consisting of hundreds or thousands of atoms. In this paper we present a method for carrying out vibrationally adiabatic mixed quantum-classical simulations in condensed phases that is accurate, efficient, and generalizable to multiple degrees-of-freedom problems. This approach is an extension of, and a significant improvement upon, our previous work that was applicable only to nonrotating solutes. ${ }^{1}$

One possible application of vibrationally adiabatic QC simulations is the study of vibrational dephasing in condensed phases. ${ }^{2-10}$ Oxtoby and co-workers have obtained the dephasing times of diatomic molecules in condensed phase environments by using perturbation theory to calculate the time-dependent fluctuations in the vibrational energy levels. ${ }^{9,10}$ A completely classical simulation was used with rigid molecules so that there was no influence of the quantum-mechanical system on the classical motion. This approach has been widely and successfully used for weakly coupled solute-solvent systems. ${ }^{9-13}$ A vibrationally adiabatic QC simulation directly provides the fluctuating frequencies of a quantum-mechanical solute from dynamics that include response from the quantum mode(s) onto the classical coor-

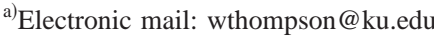

dinates. Further, an explicit calculation of the vibrational energy levels is accurate even for strong solute-solvent coupling, unlike perturbation theory approaches.

Another potential problem for which mixed quantumclassical simulations may be useful is vibrational relaxation. The most prevalent approach for calculating vibrational relaxation lifetimes in condensed phase systems is based on perturbation theory in which the relaxation rate, e.g., from $v=1$ to $v=0$, is given by ${ }^{14}$

$$
k_{0 \leftarrow 1}(T)=q(T) \int_{-\infty}^{\infty} e^{i \omega_{o} t}\langle F(t) F(0)\rangle_{\mathrm{cl}} d t,
$$

where $\omega_{o}$ is the frequency of the oscillator. The prefactor $q(T)$ ensures that detailed balance is satisfied, i.e., $k_{0 \leftarrow 1}(T)=e^{\hbar \omega_{o} / k_{b} T} k_{1 \leftarrow 0}(T)$. The classical force-force time correlation function, $\langle F(0) F(t)\rangle_{\mathrm{cl}}$, is calculated by a molecular dynamics simulation in which the oscillator is frozen at its equilibrium distance; $F$ is the force exerted along the oscillator by the solvent. Thus, the assumption is that the relaxation occurs due to the solvent friction acting on the oscillator. This approach has been used extensively, often with great success. ${ }^{15-19}$ However, it has also been established for some time that perturbation theory can fail for highfrequency oscillators in gas-phase collisions. ${ }^{20}$ More recent studies have found that the conventional perturbation theory approach $^{14}$ gives lifetimes that differ significantly from experimental measurements in both clusters $^{21}$ and liquids. ${ }^{22,23}$ This has been attributed to the potential energy surfaces ${ }^{23}$ and, more significantly, to the need to modify the conventional perturbation theory approach using quantum correction factors, ${ }^{23-26}$ i.e., by choosing an appropriate $q(T)$. However, even when such a modification successfully returns the correct rate, the perturbation theory still provides limited and indirect information about how the energy is deposited in the surroundings and about competition between intramolecular 
vibrational redistribution and vibrational energy transfer. These issues cannot be addressed directly in the perturbation theory approach since the dynamics of the relaxation event itself are not simulated.

The applicability of mixed QC approaches to calculate vibrational relaxation rate constants (and other quantities) within the Golden Rule formalism has been called into question by Berne and co-workers. ${ }^{25,27}$ However, they have focused, in addition to perturbation theory approaches, on special systems for which quantum mechanical solutions are available. These typically involve a harmonic bath and linear, bi-linear, or exponential coupling. The ability to carry out vibrationally adiabatic mixed QC simulations allows for a wider examination of these issues including consideration of (1) systems with arbitrary (anharmonic) vibrational potentials and solute-solvent coupling, (2) methods that go beyond perturbation theory approaches to calculating the rate constant, (3) comparisons of vibrationally diabatic and adiabatic approaches, and (4) vibrational quantum state dependence of relaxation lifetimes. In these cases, testing QC methods by comparison with rigorous results can be complicated by the absence of quantum-mechanical calculations; comparisons with experimental data and calculations involving systematic approximations must be used instead.

One approach, suggested by Tully, ${ }^{28}$ that moves beyond the perturbation theory approximation and allows insight into the basic mechanism of vibrational relaxation is a direct calculation of the vibrational relaxation rate constant by a surface hopping simulation. Many approaches for incorporating nonadiabatic dynamics have been developed and could be applied to this problem..$^{28-35}$ The approach presented here is applicable to this problem (for cases when the vibrational relaxation lifetime is not too long). Several studies have used a surface-hopping approach with vibrationally diabatic states $^{36,37}$ and one group calculated vibrational relaxation lifetimes using a mean-field approach based on diabatic states. $^{38}$

The organization of the remainder of the paper is as follows: Mixed quantum-classical equations of motion for a solute with a quantum mechanical vibration in a classical solvent are reviewed in Sec. II. A suitable molecular dynamics algorithm for integrating these equations is given in the Appendix. The approach for solving the vibrational Schrödinger equation at each time step in a mixed quantumclassical simulation is described in Secs. III and IV. Results are presented and discussed in Sec. V. Finally, some concluding remarks are offered in Sec. VI.

\section{EQUATIONS OF MOTION}

The mixed quantum-classical approach we consider is simply the standard Born-Oppenheimer approximation, ${ }^{39}$ but applied between a fast vibrational coordinate and slow rotational and translational degrees-of-freedom. Vibrationally adiabatic approaches have been used to investigate a number of systems including proton transfer reactions, ${ }^{34,35,40-42}$ vibrational relaxation, ${ }^{43}$ and vibrationvibration energy transfer. ${ }^{44}$ They are related to the mixed quantum-classical time-dependent self-consistent field or Ehrenfest approaches, ${ }^{30,45-51}$ but with important differences. ${ }^{29}$ As discussed in the Introduction, vibrationally adiabatic dynamics can be generalized by incorporating nonadiabatic transitions. ${ }^{28-35}$

In this section, we review the mixed quantum-classical equations of motion for a single diatomic solute molecule with a quantum-mechanical vibration and classical translation and rotation dissolved in a solvent of classical atoms. (The extension to a molecular solvent is straightforward.) We begin with a purely classical Hamiltonian and "quantize" the vibrational coordinate. The classical Hamiltonian for this system can be written as

$$
\begin{aligned}
H\left(r, p_{r}, \mathbf{e}, \mathbf{p}_{e}, \mathbf{Q}, \mathbf{P}\right)= & \frac{p_{r}^{2}}{2 \mu}+\frac{\mathbf{p}_{e}^{2}}{2 \mu r^{2}}+\sum_{j=1}^{N} \frac{\mathbf{P}_{j}^{2}}{2 m_{j}} \\
& +V(r, \mathbf{e}, \mathbf{Q}),
\end{aligned}
$$

where $r$ is the diatom bond distance, e is a Cartesian unit vector $\mathbf{e}=\left(e_{x}, e_{y}, e_{z}\right)$ pointing along the diatom bond such that $\mathbf{r}=r \mathbf{e}$, and $\mathbf{Q}=\left(\mathbf{Q}_{1}, \mathbf{Q}_{2}, \ldots, \mathbf{Q}_{N}\right)$ are the positions of the solute center-of-mass and the $N-1$ rare gas atoms. The orientational vector $\mathbf{e}$ is subject to the constraint $\mathbf{e} \cdot \mathbf{e}=1$. The conjugate momenta to these coordinates are $p_{r}, \mathbf{p}_{e}$ $=\left(p_{e_{x}}, p_{e_{y}}, p_{e_{z}}\right)$, and $\mathbf{P}=\left(\mathbf{P}_{1}, \mathbf{P}_{2}, \ldots, \mathbf{P}_{N}\right)$.

At this point we wish to treat the diatom bond distance quantum mechanically while retaining a classical description for all the other degrees-of-freedom. Specifically, we can define a quantum-mechanical Hamiltonian operator in $r$ that depends parametrically on $\mathbf{e}, \mathbf{p}_{e}$, and $\mathbf{Q}$ as

$$
\hat{h}_{r}\left(\mathbf{e}, \mathbf{p}_{e}, \mathbf{Q}\right)=\frac{\hat{p}_{r}^{2}}{2 \mu}+V_{\mathrm{eff}}\left(\hat{r} ; \mathbf{e}, \mathbf{p}_{e}, \mathbf{Q}\right),
$$

where the effective potential is

$$
V_{\mathrm{eff}}\left(\hat{r} ; \mathbf{e}, \mathbf{p}_{e}, \mathbf{Q}\right)=\frac{\mathbf{p}_{e}^{2}}{2 \mu r^{2}}+V(\hat{r}, \mathbf{e}, \mathbf{Q}) .
$$

Note that $V_{\text {eff }}$ and $\hat{h}_{r}$ are implicit functions of time since e, $\mathbf{p}_{e}$, and $\mathbf{Q}$ are classical variables dependent on time. The adiabatic vibrational states are then obtained by solving the Schrödinger equation for fixed $\mathbf{e}, \mathbf{p}_{e}$, and $\mathbf{Q}$

$$
\hat{h}_{r}\left(\mathbf{e}, \mathbf{p}_{e}, \mathbf{Q}\right) \phi_{n}\left(r ; \mathbf{e}, \mathbf{p}_{e}, \mathbf{Q}\right)=E_{n}\left(\mathbf{e}, \mathbf{p}_{e}, \mathbf{Q}\right) \phi_{n}\left(r ; \mathbf{e}, \mathbf{p}_{e}, \mathbf{Q}\right) .
$$

The classical Hamiltonian for the remaining degrees-offreedom, indexed by the vibrational quantum number, can be taken as

$$
\begin{aligned}
H_{n}\left(\mathbf{e}, \mathbf{p}_{e}, \mathbf{Q}, \mathbf{P}\right) & =\sum_{j=1}^{N} \frac{\mathbf{P}_{j}^{2}}{2 m_{j}}+\left\langle\phi_{n}\left|\hat{h}_{r}\right| \phi_{n}\right\rangle_{r} \\
& =\sum_{j=1}^{N} \frac{\mathbf{P}_{j}^{2}}{2 m_{j}}+E_{n}\left(\mathbf{e}, \mathbf{p}_{e}, \mathbf{Q}\right),
\end{aligned}
$$

where the subscript $r$ indicates integration over only this coordinate. In order to ensure that the directional vector $\mathbf{e}$ is normalized we introduce a Lagrange multiplier, ${ }^{52}$ so that

$$
H_{n}\left(\mathbf{e}, \mathbf{p}_{e}, \mathbf{Q}, \mathbf{P}\right)=\sum_{j=1}^{N} \frac{\mathbf{P}_{j}^{2}}{2 m_{j}}+E_{n}\left(\mathbf{e}, \mathbf{p}_{e}, \mathbf{Q}\right)+\lambda(\mathbf{e} \cdot \mathbf{e}-1)
$$


The resulting classical equations of motion are then given by

$$
\dot{e}_{\alpha}=\frac{\partial H_{n}}{\partial p_{e_{\alpha}}} \quad \dot{p}_{e_{\alpha}}=-\frac{\partial H_{n}}{\partial e_{\alpha}}
$$

and

$$
\dot{Q}_{j \alpha}=\frac{\partial H_{n}}{\partial P_{j \alpha}} \quad \dot{P}_{j \alpha}=-\frac{\partial H_{n}}{\partial Q_{j \alpha}},
$$

where $\alpha=x, y, z$ and $j=1,2, \ldots N$.

In this QC formulation the vibrationally adiabatic eigenfunctions are known and the forces can be calculated by the Hellmann-Feynman theorem. ${ }^{53}$ For example,

$$
\begin{aligned}
f_{j \alpha}^{(n)}\left(\mathbf{e}, \mathbf{p}_{e}, \mathbf{Q}\right) & =-\frac{\partial H_{n}}{\partial Q_{j \alpha}} \\
& =-\frac{\partial E_{n}(\mathbf{Q})}{\partial Q_{j \alpha}} \\
& =-\left\langle\phi_{n}\left|\frac{\partial V(\hat{r}, \mathbf{e}, \mathbf{Q})}{\partial Q_{j \alpha}}\right| \phi_{n}\right\rangle_{r},
\end{aligned}
$$

is the force in the $\alpha$-direction on the $j$ th solvent atom with the solute in vibrational state $n$. For the directional vector components, there is an additional component proportional to the Lagrange multiplier

$$
\begin{aligned}
f_{e_{\alpha}}^{(n)}\left(\mathbf{e}, \mathbf{p}_{e}, \mathbf{Q}\right) & =-\left\langle\phi_{n}\left|\frac{\partial V(\hat{r}, \mathbf{e}, \mathbf{Q})}{\partial e_{\alpha}}\right| \phi_{n}\right\rangle_{r}-2 \lambda e_{\alpha} \\
& \equiv \widetilde{f}_{e_{\alpha}}^{(n)}-2 \lambda e_{\alpha} .
\end{aligned}
$$

Note that we have chosen to include the rotational kineticenergy term, $\mathbf{p}_{e}^{2} /\left(2 \mu r^{2}\right)$, in the effective potential, Eq. (2.3). This is certainly not the only possible approach but it has the advantage that all $r$-dependent terms are included in the vibrational Schrödinger equation, in this case the centrifugal potential is incorporated. Hence, an eigenfunction calculated in this approach should be closer to the true eigenfunction, i.e., that obtained from a fully quantum-mechanical calculation, along the $r$ coordinate. This means, however, that evaluating $\partial H_{n} / \partial p_{e_{\alpha}}$ requires some consideration. Ignoring the action of the orientational kinetic energy on the adiabatic eigenfunctions, the Hellmann-Feynman theorem gives

$$
\begin{aligned}
g_{e_{\alpha}}\left(\mathbf{e}, \mathbf{p}_{e}, \mathbf{Q}\right) & =\frac{\partial H_{n}}{\partial p_{e_{\alpha}}}=\left\langle\phi_{n}\left|\frac{\partial \hat{h}_{r}}{\partial p_{e_{\alpha}}}\right| \phi_{n}\right\rangle_{r} \\
& =\left\langle\phi_{n}\left|\frac{1}{\mu r^{2}}\right| \phi_{n}\right\rangle_{r} p_{e_{\alpha}} \equiv B^{(n)} p_{e_{\alpha}} .
\end{aligned}
$$

Thus, the equations of motion involve an effective rotational constant, $B^{(n)}$, obtained from an average over the vibrational eigenfunction. Finally, for the solvent atom coordinates, we have simply

$$
g_{j \alpha}=\frac{\partial H_{n}}{\partial P_{j \alpha}}=\frac{P_{j \alpha}}{m_{j}} .
$$

In the Appendix a rigorous molecular dynamics algorithm for integrating these mixed quantum-classical equations of motion is developed. The QC dynamics simulations results presented in Sec. V use this method.

\section{LANCZOS ALGORITHM}

In the mixed quantum-classical adiabatic molecular dynamics simulation described in Sec. VA the vibrational states of the diatomic solute must be calculated at each time step and the Hellmann-Feynman forces evaluated. Thus, a method is needed to solve the vibrational Schrödinger equation that is efficient, since it is used repeatedly, and sufficiently accurate to obtain the required forces. Furthermore, the approach should ideally be applicable to multidimensional vibrational problems. However, we can take advantage of the fact that only a few of the vibrational states are usually of interest and that the vibrational potential, and hence the eigenstates, do not change much from one time step to the next. Given these considerations, we use the iterative Lanczos algorithm ${ }^{54}$ with full reorthogonalization and a special choice of the starting vector to obtain the vibrational eigenvalues and eigenfunctions. Direct diagonalization of the vibrational Hamiltonian would suffice for this one degree-offreedom problem, however, the present approach should be applicable to vibrational problems involving multiple degrees-of-freedom.

Briefly, in the Lanczos scheme an initial vector in the basis $\mathbf{v}_{0}$ (usually taken as random) is used to build a smaller $(M \ll N)$ Krylov space basis set by repeated application of the Hamiltonian matrix:

$$
\left\{\mathbf{v}_{0}, \mathbf{H} \cdot \mathbf{v}_{0}, \mathbf{H}^{2} \cdot \mathbf{v}_{0}, \mathbf{H}^{3} \cdot \mathbf{v}_{0}, \ldots, \mathbf{H}^{M-1} \cdot \mathbf{v}_{0}\right\},
$$

where each vector is Gram-Schmidt orthogonalized against all previous vectors. Full orthogonalization of the Krylov vectors is not costly for the small number of vectors required for the present applications and it allows us to obtain the eigenfunctions, which are required to calculate the Hellmann-Feynman forces, in addition to the eigenvalues. The Hamiltonian matrix in this Krylov basis is then diagonalized to obtain the eigenvalues.

This approach has several advantages for vibrationally adiabatic dynamics: (1) It is efficient. The lowest energy eigenstates, those that are of interest in the study of vibrational spectra, are converged rapidly. The fewer vibrational states that are required, the lower the computational effort. It will be seen in Sec. V that simulations involving the solution of the vibrational Schrödinger equation millions of times are possible with the present approach. (2) It is tunable. The desired accuracy in the eigenvalues can be specified and the computational effort scales accordingly. (3) The result at one time step can be used to accelerate the calculation at the next time step. Because the vibrational potential (and hence eigenfunctions) change little between time steps, the eigenfunctions obtained at time $t$ can be used to initiate the Lanczos scheme at time $t+\delta t$ by using the starting vector

$$
\mathbf{v}_{0}(t+\delta t)=\frac{1}{N_{\text {states }}} \sum_{n=1}^{N_{\text {states }}} \phi_{n}(t),
$$

where $N_{\text {states }}$ is the number of vibrational states of interest. 


\section{POTENTIAL-OPTIMIZED DVR}

As emphasized in Sec. III, it is critically important to optimize the efficiency of the calculation of the diatom vibrational states since it must be repeated at least once every time step and hence thousands or millions of times during a simulation. One approach to this problem is to reduce the size of the basis set as much as possible without sacrificing accuracy. For the one-dimensional vibrational system considered here the basis set size is significantly reduced by using a potential-optimized discrete variable representation ${ }^{55,56}$ (PODVR). This is an improvement upon our previous work using a sinc-function DVR basis. ${ }^{1}$ Originally, PO-DVRs were developed to optimize the one-dimensional bases used in solving multidimensional vibrational problems with direct product basis sets. Here we use a PO-DVR for a different purpose: to optimize the one-dimensional basis for repeated solution of the Schrödinger equation. The approach is as follows.

A "raw" basis is chosen, consisting of a large number of basis functions. We choose a $(0$ to $\infty)$ sinc-function ${ }^{57}$ discrete variable representation ${ }^{58,59}$ as the raw basis set. In this basis, closed form analytical expressions for the kinetic energy matrix elements are available $e^{57}$ and the potential energy matrix is approximated as diagonal, with each matrix element equal to the potential energy at the corresponding grid point. The Hamiltonian matrix in this basis, $\mathbf{H}^{\mathrm{RAW}}$, is thus easily evaluated. It is necessary to choose a reference potential, the one for which the PO-DVR basis will be optimized. We simply take the vibrational potential for the diatomic molecule at the first step of the QC simulation. There is no reason to believe that this is the optimum choice but in applications to this point it has worked quite well.

Once the Hamiltonian matrix in the raw basis is calculated, the vibrational Schrödinger equation

$$
\mathbf{H}^{\mathrm{RAW}} \cdot \psi_{n}=E_{n} \psi_{n}, \quad n=1,2, \ldots, \mathcal{N}_{\mathrm{RAW}},
$$

is solved for the energy eigenvalues, $E_{n}$, and eigenvectors, $\psi_{n}$. The eigenvectors corresponding to the lowest $\mathcal{N}_{\text {PO }}$ energy eigenvalues are then used as a new, smaller basis in which the matrix of the position operator can be calculated. That is, if the raw basis functions are denoted by $\left\{\left|\varphi_{j}\right\rangle\right\}_{j=1}^{\mathcal{N}_{\text {RAW }}}$ with DVR grid points $r_{j}$, the solution of the Schrödinger equation yields the coefficients $c_{j}^{n}$ defined by

$$
\left|\psi_{n}\right\rangle=\sum_{j=1}^{\mathcal{N}_{\mathrm{RAW}}} c_{j}^{n}\left|\varphi_{j}\right\rangle
$$

Then, the matrix elements of the position operator, $\hat{r}$, can be calculated by

$$
(\mathbf{r})_{k, l}=\left\langle\psi_{k}|\hat{r}| \psi_{l}\right\rangle=\sum_{j=1}^{\mathcal{N}_{\mathrm{RAW}}} c_{j}^{k} c_{j}^{l} r_{j}, \quad k, l=1,2, \ldots, \mathcal{N}_{\mathrm{PO}},
$$

where we have assumed that the coefficients $c_{j}^{n}$ are real. Note that $\mathcal{N}_{\text {PO }}<\mathcal{N}_{\text {RAW }}$ (or ideally $\mathcal{N}_{\text {PO }} \ll \mathcal{N}_{\text {RAW }}$ ) so that this matrix representation of $\hat{r}$ involves a significantly smaller basis than the raw one.

The position matrix is then diagonalized,

$$
\mathbf{r} \cdot \chi_{\alpha}=s_{\alpha} \chi_{\alpha}, \quad \alpha=1,2, \ldots, \mathcal{N}_{\mathrm{PO}},
$$

giving the $\mathcal{N}_{\mathrm{PO}}$ eigenvalues $s_{\alpha}$ which are the grid points in the PO-DVR and $\mathcal{N}_{\mathrm{PO}}$ corresponding eigenvectors $\chi_{\alpha}$ which are the PO-DVR basis functions. As usual, the potential energy matrix in the PO-DVR is approximated as diagonal, with the matrix elements $\left(\mathbf{V}^{\mathrm{PO}}\right)_{\alpha, \beta}=V\left(r=s_{\alpha}\right) \delta_{\alpha, \beta}$. The kinetic-energy matrix can be obtained by transforming the matrix in the raw basis, so that

$$
\begin{aligned}
\left(\mathbf{T}^{\mathrm{PO}}\right)_{\alpha, \beta}= & \left\langle\chi_{\alpha}|\hat{T}| \chi_{\beta}\right\rangle=\sum_{j=1}^{\mathcal{N}_{\text {RAW }}} \sum_{i=1}^{\mathcal{N}_{\text {RAW }}}\left\langle\chi_{\alpha} \mid \varphi_{j}\right\rangle\left\langle\varphi_{j}|\hat{T}| \varphi_{i}\right\rangle \\
& \times\left\langle\varphi_{i} \mid \chi_{\beta}\right\rangle,
\end{aligned}
$$

invoking a completeness relation for the sinc-function DVR basis. Note that the kinetic-energy matrix in the raw basis, $\left\langle\varphi_{j}|\hat{T}| \varphi_{i}\right\rangle$, is already calculated and, since from the diagonalization of $\mathbf{r}$ we obtain

$$
\left|\chi_{\alpha}\right\rangle=\sum_{k=1}^{\mathcal{N}_{\mathrm{PO}}} b_{k}^{\alpha}\left|\psi_{k}\right\rangle
$$

we have

$$
\left\langle\varphi_{j} \mid \chi_{\alpha}\right\rangle=\sum_{k=1}^{\mathcal{N}_{\mathrm{PO}}} b_{k}^{\alpha}\left\langle\varphi_{j} \mid \psi_{k}\right\rangle=\sum_{k=1}^{\mathcal{N}_{\mathrm{PO}}} b_{k}^{\alpha} c_{j}^{k},
$$

from Eq. (4.2). Thus, everything necessary for calculating the kinetic-energy matrix in the PO-DVR basis is already in hand.

The use of the PO-DVR reduces the size of the Hamiltonian matrix that must be diagonalized to obtain the vibrational energy levels and eigenfunctions. In addition, it improves the efficiency of the calculation by significantly reducing the number of potential evaluations (from $\mathcal{N}_{\text {RAW }}$ to $\mathcal{N}_{\mathrm{PO}}$ ). This may, in some circumstances, represent the greater savings. One of the important benefits of this approach is that it can be straightforwardly applied to multidimensional systems such as polyatomic molecules. Furthermore, as described here the PO-DVR is optimized for the lowest energy states and thus may be tuned to fit the number of energy states of interest.

\section{SIMULATION RESULTS}

\section{A. Simulation details}

Except where otherwise indicated, the simulations were carried out with a single $\mathrm{N}_{2}$ solute molecule and 255 argon solvent atoms in a cubic box of length $22.92 \AA$ (giving a density of $1.41 \mathrm{~g} / \mathrm{cm}^{3}$ ) with periodic boundary conditions. The interaction potential is taken to be a sum of pairwise terms. The $\mathrm{N}_{2}$ potential is taken to be a Morse function with $D=9.755 \mathrm{eV}, \alpha=2.75 \AA^{-1}$, and $r_{e}=1.094 \AA$. The remaining interatomic potentials are of Lennard-Jones form, with $\epsilon_{\mathrm{N}}=37.3 \mathrm{~K}, \quad \sigma_{\mathrm{N}}=3.31 \AA,{ }^{9} \quad \epsilon_{\mathrm{Ar}}=124.96 \mathrm{~K}, \quad$ and $\sigma_{\mathrm{Ar}}$ $=3.42 \AA$. The usual combination formulas are used, e.g., $\epsilon_{\mathrm{N}-\mathrm{Ar}}=\sqrt{\epsilon_{\mathrm{N}} \epsilon_{\mathrm{Ar}}}$ and $\sigma_{\mathrm{N}-\mathrm{Ar}}=\left(\sigma_{\mathrm{N}}+\sigma_{\mathrm{Ar}}\right) / 2$. The interactions are truncated at a radius of $11 \AA$.

The simulations are initiated from an FCC lattice. The system is propagated under completely classical dynamics (with a frozen $\mathrm{N}_{2}$ bond distance) for $25 \mathrm{ps}$ (with a time step 


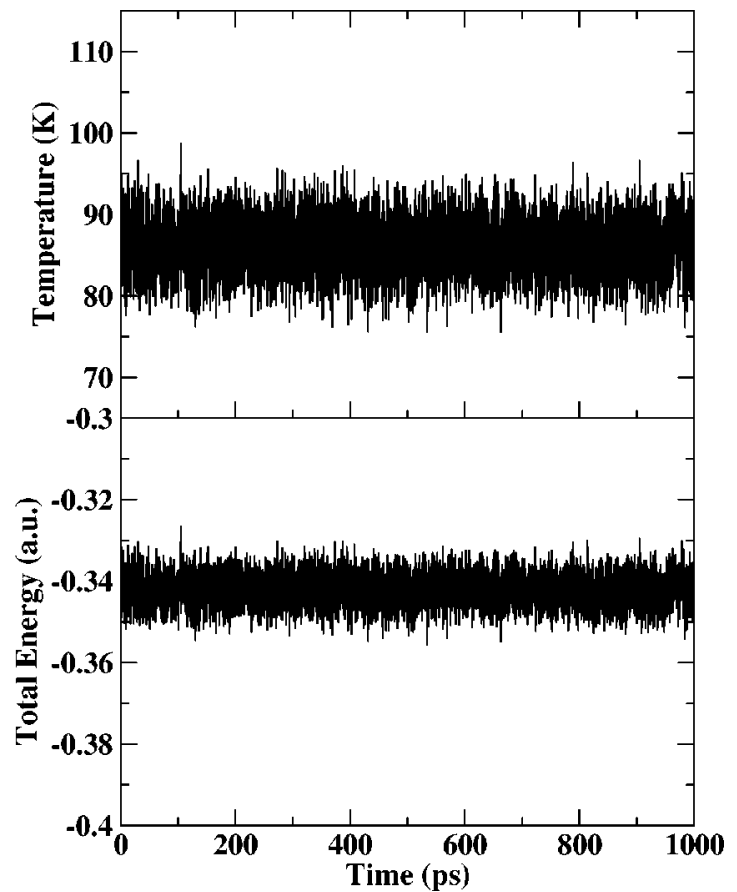

FIG. 1. The instantaneous temperature and total energy are shown as a function of time for a mixed quantum-classical simulation of $\mathrm{N}_{2}(n=0)$ in liquid Ar.

of $\delta t=2.5 \mathrm{fs})$ at $T=300 \mathrm{~K}$. The system is then equilibrated under the mixed quantum-classical dynamics for $10 \mathrm{ps}(\delta t$ $=2 \mathrm{fs}$ ). In the classical and QC equilibration dynamics, the velocities are rescaled to keep the temperature within $\pm 10 \mathrm{~K}$ of the desired value for the first 12.5 and $5 \mathrm{ps}$, respectively. The dynamics are then propagated in an NVE ensemble during which the data is collected for analysis.

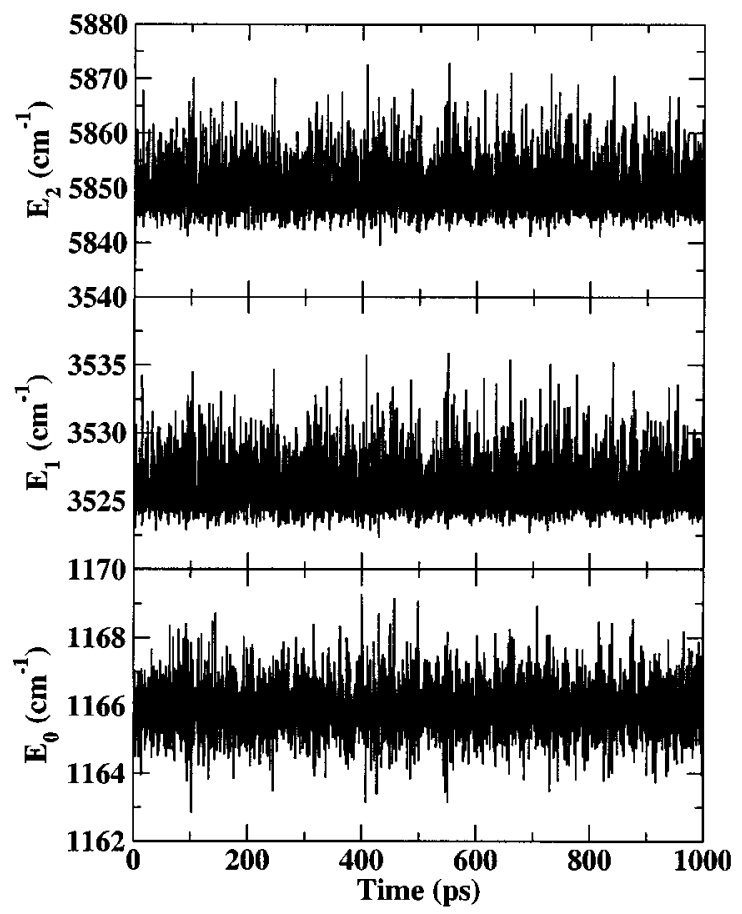

The sinc-function discrete variable representation, the "raw" basis used (see Sec. IV), has evenly spaced grid points, ${ }^{57}$ here the spacing is $\Delta q=0.01079 \AA$. An energy cutoff is used in which all grid points at which the potential energy (relative to the minimum) is greater than $V_{\text {cut }}=5 \mathrm{eV}$ are discarded. The resulting grid has 60 points. The number of PO-DVR basis functions is taken as input.

The convergence of the Lanczos algorithm is determined by monitoring the $n=3$ vibrational level; if the fractional change between Lanczos iterations $M$ and $M+1$ in this eigenvalue, $\delta=\left(E_{3}^{M+1}-E_{3}^{M}\right) / E_{3}^{M+1}$, is less than $1 \times 10^{-10}$ the eigenvalue calculation is stopped.

The instantaneous temperature and total energy are plotted as a function of time in Fig. 1 for a run of $1 \mathrm{~ns}(\delta t$ $=2 \mathrm{fs}$ ) using 15 PO-DVR basis functions. The average temperature during the run is $86.3 \mathrm{~K}$. Note that the total energy displays little drift (a linear fit to the total energy gives a slope of $2.4539 \times 10^{-7}$ a.u./ps). The molecular dynamics integration algorithm given in the Appendix may not be optimally efficient, however, this demonstrates that long-time dynamics are accessible and that the method is stable. For reference, with a time step of $2 \mathrm{fs}$ this simulation involved the calculation of the $\mathrm{N}_{2}$ vibrational states $\sim 1.5 \times 10^{6}$ times.

\section{B. Adiabatic energy levels}

The three lowest vibrationally adiabatic energy levels of $\mathrm{N}_{2}$ are shown as a function of time in Fig. 2 for the $1 \mathrm{~ns}$ simulation of $\mathrm{N}_{2}(n=0)$ in liquid Ar described in Sec. V A. Plots of the energies are given over both the entire run time of the simulation and a 15 ps interval.

We focus first on the gross features of the energy levels over the entire simulation. It is immediately apparent that the

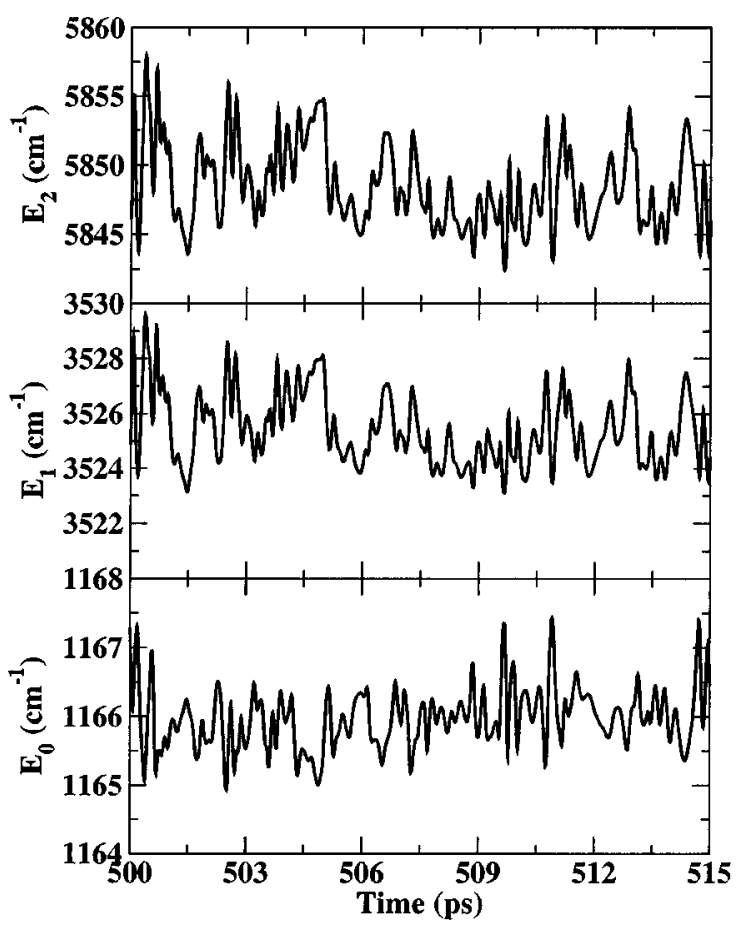

FIG. 2. The vibrationally adiabatic energy levels of $\mathrm{N}_{2}$ (quantum numbers $n=0,1,2$ ) are shown as a function of time for a mixed quantum-classical simulation of $\mathrm{N}_{2}(n=0)$ in liquid Ar. Results are presented for the entire 1 ns simulation run and a 15 ps time interval in the middle of the run. 
scale of the energy level fluctuations increases with the vibrational quantum number. The $n=0$ vibrational energy modulations span a range of $6.5 \mathrm{~cm}^{-1}$, compared with 13.8 and $33.8 \mathrm{~cm}^{-1}$ for the $n=1$ and $n=2$ levels, respectively. Thus, the influence of the Ar solvent on the vibrational energy increases with the vibrational quantum number $n$. This can be attributed to the larger effective size of the $\mathrm{N}_{2}$ molecule in the vibrationally excited states. Another feature of the energy level fluctuations is the asymmetry about the mean. The $n=0$ energy level fluctuations are roughly symmetric about the mean energy. In contrast, the $n=1$ and 2 energy levels exhibit significant asymmetry with fluctuations to higher vibrational energies predominating. This difference between the $n=0$ and $n>0$ vibrational states reflects the fact that the solvent is interacting with the $n=0$ vibrational state. The energies of the higher vibrational states, which correspond to an effectively larger $\mathrm{N}_{2}$ molecule, are more likely to be shifted to higher energies by these interactions.

Using the calculated energy levels presented in Fig. 2, the average $\mathrm{N}_{2}$ fundamental frequency in the simulation is $\left\langle\omega_{01}\right\rangle=2359.8 \mathrm{~cm}^{-1}$, giving a blue-shift of $1.9 \mathrm{~cm}^{-1}$ relative to the gas phase frequency (for this potential) of $\omega_{01}$ $=2357.9 \mathrm{~cm}^{-1}$. The "hot band" average frequency is $\left\langle\omega_{12}\right\rangle=2323.5 \mathrm{~cm}^{-1}$, representing a blue-shift of $2.1 \mathrm{~cm}^{-1}$ from the gas phase value of $2321.4 \mathrm{~cm}^{-1}$. [Note that this $\left\langle\omega_{12}\right\rangle$ value is obtained from a simulation of $\mathrm{N}_{2}(n=0)$, so it is not precisely the transition frequency relevant to, e.g., the hot band in the Raman spectrum.]

A look at the details of the modulation of the vibrational energy levels is provided by the plots over the 15 ps time interval, shown in Fig. 2. Specifically, the fluctuations of the $E_{1}$ and $E_{2}$ levels caused by the Ar solvent occur relatively in phase. In contrast, the modulations of the ground state energy $E_{0}$ do not occur in phase with those of the higher vibrational states. In fact, it appears that the fluctuations in $E_{0}$ are to some degree anticorrelated with those of $E_{1}$ and $E_{2}$, i.e., $E_{0}$ peaks where $E_{1}$ and $E_{2}$ are at a minimum and vice versa. The calculated vibrational frequencies as a function of time can be used to obtain the pure dephasing times, e.g., using the theoretical approaches of $\mathrm{Kubo}^{2}$ and Oxtoby. ${ }^{9,10}$ (For systems like $\mathrm{N}_{2}$ in Ar, the energy relaxation time, $T_{1}$ is much longer than the pure dephasing time so that the total dephasing time is dominated by pure dephasing, $T_{2}=T_{2}^{*}$.) A study of dephasing times using vibrationally adiabatic QC simulations is underway and will be given elsewhere.

The normalized probability distributions of the $0 \rightarrow 1$ and $1 \rightarrow 2$ transition energies obtained from the $1 \mathrm{~ns}$ simulation at $85 \mathrm{~K}$ with $\mathcal{N}_{\mathrm{PO}}=15$ are shown in Fig. 3. The anharmonicity in the vibrational potential is evidenced by the $\sim 36 \mathrm{~cm}^{-1}$ shift of $E_{12}$ to lower energies from $E_{01}$. The two distributions are qualitatively similar. They are both asymmetric, with a single peak that decays more slowly at higher frequencies. The distribution of $E_{01}$ can be compared with that from our previous simulations of nonrotating $\mathrm{N}_{2}$ in $\mathrm{Ar}$ at $150 \mathrm{~K}$. ${ }^{1}$ An asymmetric distribution was also observed in that case; that distribution is wider with a full width at half maximum or $4.4 \mathrm{~cm}^{-1}$ compared to $3.5 \mathrm{~cm}^{-1}$ for the present case. A similar asymmetric distribution in the transition en-

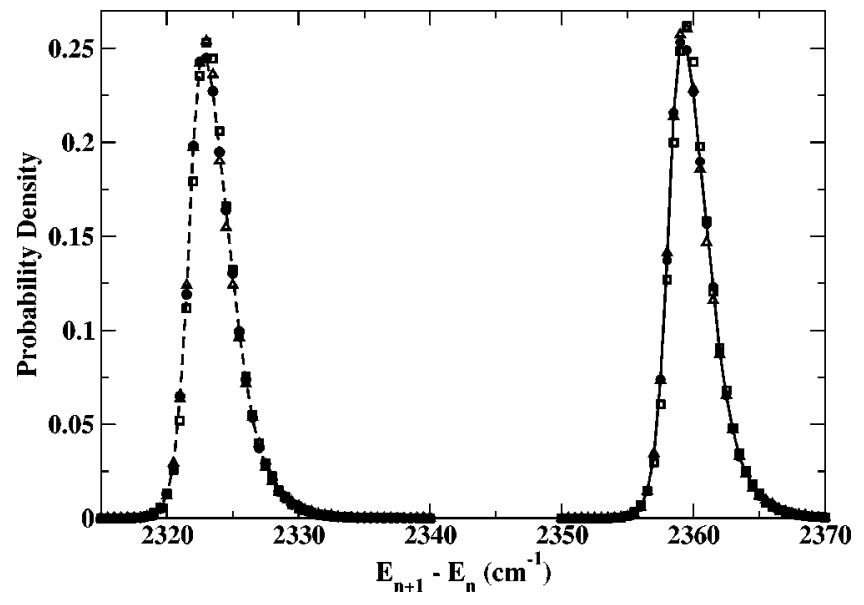

FIG. 3. The normalized distributions of the energy gaps $E_{01}$ (solid lines) and $E_{12}$ (dashed lines) are shown, obtained from the 1 ns QC simulation of $\mathrm{N}_{2}(n=0)$ in liquid Ar. Results are shown for $\mathcal{N}_{\mathrm{PO}}=10$ (squares), 12 (triangles), and 15 (circles with lines).

ergy was found by Herman and Berne in Monte Carlo simulations of $\mathrm{Br}_{2}$ in $\mathrm{Ar}^{60}$

The transition energy distributions obtained from $1 \mathrm{~ns}$ simulations at $85 \mathrm{~K}$ with $\mathcal{N}_{\mathrm{PO}}=10$ and 12 are also shown in Fig. 3 for comparison with the $\mathcal{N}_{\mathrm{PO}}=15$ results. The distributions obtained for these three sizes of the PO-DVR basis set are very similar; only minor quantitative differences are observed. This indicates that an accurate solution of the vibrational Schrödinger equation can be obtained with a basis consisting of only 10 functions (see also Sec. V D). For reference, the average frequency (blue-) shift obtained in the calculation with $\mathcal{N}_{\mathrm{PO}}=10,12$, and 15 is $1.98,1.92$, and $1.93 \mathrm{~cm}^{-1}$, respectively.

\section{Nonadiabatic coupling}

In systems for which the vibrational relaxation lifetime is sufficiently short $\left(\mathrm{N}_{2}\right.$ in Ar is not such a system) the vibrational relaxation may be explicitly simulated by a surfacehopping or classical mapping algorithm. (However, see Sec. I for a caveat.) However, these approaches require knowledge of the nonadiabatic coupling, a quantity provided by the mixed quantum-classical approach presented here. The vibrationally nonadiabatic (kinetic) coupling due to solvent atom $j$,

$$
\begin{aligned}
\mathbf{d}_{n^{\prime}, n}^{j}\left(\mathbf{e}, \mathbf{p}_{e}, \mathbf{Q}\right) & =\left\langle\phi_{n^{\prime}} \mid \frac{\partial \phi_{n}}{\partial \mathbf{Q}_{j}}\right\rangle_{r} \\
& =\frac{\left\langle\phi_{n^{\prime}}\left|\partial V\left(\hat{r} ; \mathbf{e}, \mathbf{p}_{e}, \mathbf{Q}\right) / \partial \mathbf{Q}_{j}\right| \phi_{n}\right\rangle_{r}}{E_{n}\left(\mathbf{e}, \mathbf{p}_{e}, \mathbf{Q}\right)-E_{n^{\prime}}\left(\mathbf{e}, \mathbf{p}_{e}, \mathbf{Q}\right)},
\end{aligned}
$$

can be obtained directly since the vibrational wavefunctions are explicitly and accurately calculated. In Fig. 4 the total coupling summed over all solvent atoms, given by

$$
D_{n^{\prime}, n}=\sum_{j=1}^{N} \mathbf{d}_{n^{\prime}, n}^{j} \cdot \mathbf{P}_{j} / m_{j}
$$

is plotted as a function of time in the $1 \mathrm{~ns}$ simulation at $85 \mathrm{~K}$. The coupling is roughly symmetric about zero. At the vast 


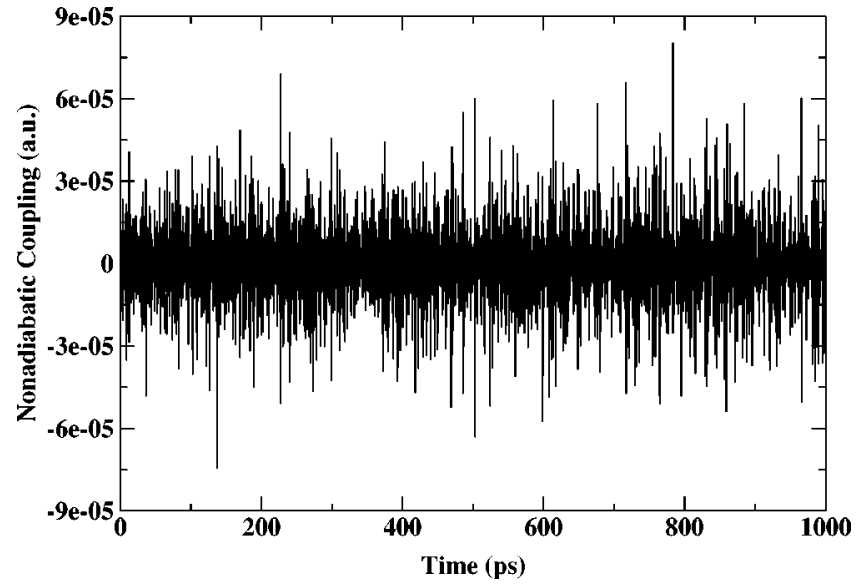

FIG. 4. The total nonadiabatic (kinetic) coupling between vibrational states $n=0$ and $n=1$ [see Eq. (5.2)].

majority of times in the trajectory, the magnitude of the coupling is small; at $\sim 84 \%(\sim 98 \%)$ of the time steps the coupling is less than or equal to one-tenth (one-fourth) the largest coupling value. Relatively large values of the coupling are reached infrequently. The distribution of coupling values may be useful in developing simple models of vibrational relaxation in condensed phases.

Note that since this coupling is responsible for vibrational relaxation, these simulations can provide insight into the solute-solvent configurations and motions that lead to the greatest probability of vibrational relaxation. Specifically, the results of these simulations include the solvent atom positions and momenta and the solute vibrational wave function as a function of time. Thus, it may be possible to gain a greater understanding of the origin of large nonadiabatic coupling (as well as the modulations of the energy levels) by examination of these mixed quantum-classical trajectories, leading to insight into the molecular-level mechanisms of dephasing and vibrational relaxation. This analysis is currently underway.

\section{Accuracy and efficiency}

The absolute value of the percent error in the first three transition energies, $E_{01}, E_{12}$, and $E_{23}$ are shown in Fig. 5 as a function of time for a 100 ps simulation of $\mathrm{N}_{2}(n=0)$ at 85 $\mathrm{K}$ with $\mathcal{N}_{\mathrm{PO}}=15$. In this simulation the vibrational eigenvalues were recalculated every 200 time steps (400 fs) using the "raw" sinc-function DVR basis $\left(\mathcal{N}_{\mathrm{SDVR}}=60\right)$ and direct diagonalization. The resulting transition energies were taken as the "exact" values for calculating the error. The error is extremely small for all the transition energies but increases by a factor of $\sim 10$ for each increment in the vibrational quantum number $n$. All the transition energies exhibit sharp peaks in the error, five appear in this time interval. However, the error in any transition energy is never larger than 1.5 $\times 10^{-6} \%$. (For reference, the errors in the $E_{01}, E_{12}$, and $E_{23}$ transition energies with $\mathcal{N}_{\mathrm{PO}}=10$, not shown, are less than $8 \times 10^{-7} \%, \quad 3 \times 10^{-6} \%$, and $9 \times 10^{-5} \%$, respectively.) Thus, the PO-DVR and Lanczos scheme provide a very accurate solution of the vibrational Schrödinger equation in these simulations.

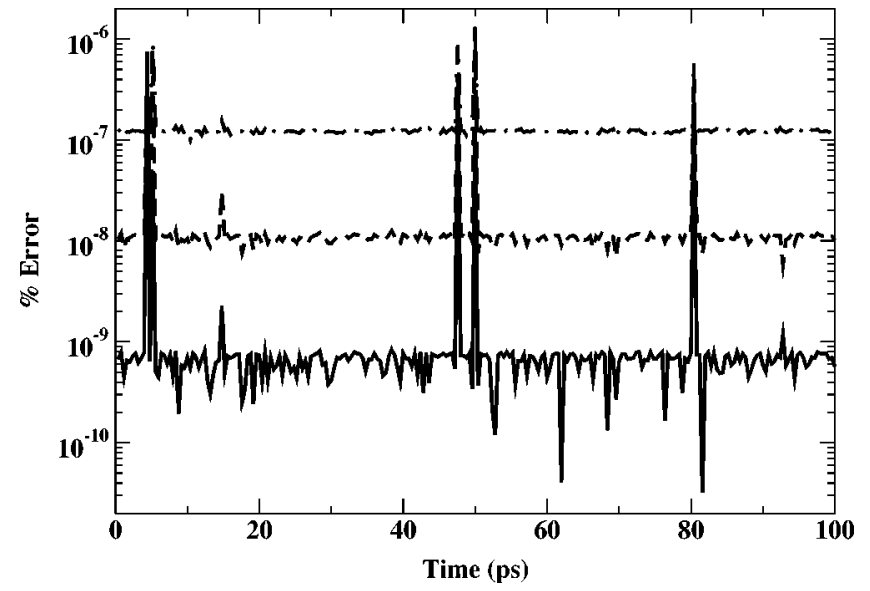

FIG. 5. The percent error (see text) in the transition energies, $E_{01}$ (solid line), $E_{12}$ (dashed line), and $E_{23}$ (dot-dashed line) for a simulation of $\mathrm{N}_{2}(n=0)$ in liquid Ar.

The key measure of the computational effort in solving the vibrational Schrödinger equation is the number of Lanczos iterations required to converge the eigenvalues. In the 1 ns simulation of $\mathrm{N}_{2}(n=0)$ at $85 \mathrm{~K}$ with $\mathcal{N}_{\mathrm{PO}}=15$ the average number of Lanczos iterations required was 6.1 and the largest number of iterations at any step was 8 . For the onedimensional vibrational problem examined here the Hamiltonian matrix is not sparse as it would be for a system with multiple degrees-of-freedom. Thus, no sparsity of the Hamiltonian matrix is exploited even though the iterative Lanczos algorithm is designed to take advantage of it (and a DVR basis in multiple degrees-of-freedom provides it). This feature of the present approach means that the extension to triatomic and polyatomic (with approximations) solute molecules is straightforward and feasible. While solution of the present one degree-of-freedom problem by direct diagonalization of the vibrational Hamiltonian matrix is possible, it will be inefficient and likely not feasible for a multiple degree-of-freedom vibrational problem. This is a key motivation for the present approach. Testing of this method for multiple degrees-of-freedom vibrational problems is currently underway.

\section{SUMMARY}

An accurate, efficient, generalizable method for carrying out mixed quantum-classical dynamics is presented. Specifically, a Lanczos scheme, with careful choice of the starting vector, is used to calculate the vibrationally adiabatic energy levels at each step in a QC trajectory. The basis set size is reduced by using a potential optimized discrete variable representation. Furthermore, a stable molecular dynamics algorithm is presented for integrating the mixed quantumclassical equations of motion.

This approach is tested on the problem of an $\mathrm{N}_{2}$ molecule with a quantum-mechanical vibration and classical translation and rotation dissolved in a classical Ar solvent. The QC molecular dynamics were simulated for $1 \mathrm{~ns}$ and the adiabatic vibrational energy levels and vibrationally nonadiabatic coupling calculated as a function of time. These quantities are important in studies of (pure) vibrational dephasing 
and vibrational relaxation. The mixed quantum-classical method presented here may be applied not only to study these problems but also spectroscopy $2,8,10,11,13$ and reaction dynamics $^{34,35,40-42}$ in condensed phase systems. A key advantage is the physical insight available from the QC trajectory consisting of solvent positions and momenta and the vibrational wave function as a function of time. In addition, it can be straightforwardly applied to solutes with multiple vibrational degrees-of-freedom.

\section{ACKNOWLEDGMENTS}

The author thanks Professor Brian B. Laird for several useful discussions. The computer facilities of the Kansas Center for Advanced Scientific Computing were used to carry out some of the calculations. Acknowledgment is made to the donors of The Petroleum Research Fund, administered by the ACS, for support of this research.

\section{APPENDIX: MOLECULAR DYNAMICS ALGORITHM}

Standard molecular dynamics algorithms, e.g., the Verlet integrator, cannot be straightforwardly applied to the classical Hamiltonian in Eq. (2.6) due to the presence of the $\mathbf{p}_{e}^{2} /\left(2 \mu r^{2}\right)$ term; the Hamiltonian involves terms mixed in the momenta and coordinates. There are a number of approaches for obtaining a stable integration algorithm, here we present one based on the implicit Euler method. ${ }^{61}$ For equations of motion given by

$$
\dot{q}=g(p, q) \quad \dot{p}=f(p, q),
$$

the Euler method is given by ${ }^{61,62}$

$$
\begin{aligned}
& q_{t+\delta t}=q_{t}+\delta \operatorname{tg}\left[p_{t}, q_{t+\delta t}\right], \\
& p_{t+\delta t}=p_{t}+\delta t f\left[p_{t}, q_{t+\delta t}\right],
\end{aligned}
$$

and also has an associated adjoint

$$
\begin{aligned}
& p_{t+\delta t}=p_{t}+\delta t f\left[p_{t+\delta t}, q_{t}\right], \\
& q_{t+\delta t}=q_{t}+\delta t g\left[p_{t+\delta t}, q_{t}\right] .
\end{aligned}
$$

An algorithm can be obtained by combining the Euler method for $t \rightarrow t+\delta t / 2$ and its adjoint for $t+\delta t / 2 \rightarrow t+\delta t$. This gives

$$
\begin{aligned}
& q_{t+\delta t / 2}=q_{t}+\frac{\delta t}{2} g\left[p_{t}, q_{t+\delta t / 2}\right], \\
& p_{t+\delta t}=p_{t}+\frac{\delta t}{2}\left\{f\left[p_{t}, q_{t+\delta t / 2}\right]+f\left[p_{t+\delta t}, q_{t+\delta t / 2}\right]\right\}, \\
& q_{t+\delta t}=q_{t+\delta t / 2}+\frac{\delta t}{2} g\left[p_{t+\delta t}, q_{t+\delta t / 2}\right] .
\end{aligned}
$$

In this case, from Eqs. (2.7)-(2.12) this algorithm gives the following procedure (suppressing the vibrational state label). First, advance the coordinates from $t$ to $t+\delta t / 2$

$$
\begin{aligned}
& Q_{j \alpha}(t+\delta t / 2)=Q_{j \alpha}(t)+\frac{\delta t}{2} \frac{P_{j \alpha}(t)}{m_{j}}, \\
& e_{\alpha}(t+\delta t / 2)=e_{\alpha}(t)+\frac{\delta t}{2} B(t+\delta t / 2) p_{e_{\alpha}}(t) .
\end{aligned}
$$

Note that Eq. (A10) must be solved self-consistently since the effective rotational constant $B(t+\delta t / 2)$ as defined in Eq. (2.11) depends on the coordinates $\mathbf{e}(t+\delta t / 2)$ and $\mathbf{Q}(t$ $+\delta t / 2)$ through the eigenfunction $\left|\phi_{n}\left(\mathbf{e}, \mathbf{p}_{e}, \mathbf{Q}\right)\right\rangle$. The solution of Eq. (A10) can be obtained by solving the vibrational Schrödinger equation at these updated positions and calculating the effective rotational constant using the resulting eigenfunctions. This provides a new estimate of $\mathbf{e}(t+\delta t / 2)$ which can be compared with the input and this procedure is repeated until the input and output $\mathbf{e}(t+\delta t / 2)$ are the same to within a specified tolerance (as measured, for example, by $\left.\Delta_{e}=\left|\mathbf{e}_{\text {output }}(t+\delta t / 2)-\mathbf{e}_{\text {input }}(t+\delta t / 2)\right|^{2}\right)$.

Next, advance the momenta a full time step from $t$ to $t$ $+\delta t$ :

$$
\begin{aligned}
& P_{j \alpha}(t+\delta t)=P_{j \alpha}(t)+\frac{\delta t}{2}\left\{f _ { j \alpha } \left[\mathbf{p}_{e}(t), \mathbf{P}(t),\right.\right. \\
& \mathbf{e}(t+\delta t / 2), \mathbf{Q}(t+\delta t / 2)]+f_{j \alpha}\left[\mathbf{p}_{e}(t+\delta t),\right. \\
& \mathbf{P}(t+\delta t), \mathbf{e}(t+\delta t / 2), \mathbf{Q}(t+\delta t / 2)]\}, \\
& p_{e_{\alpha}}(t+\delta t)=p_{e_{\alpha}}(t)+\frac{\delta t}{2}\left\{\widetilde { f } _ { e _ { \alpha } } \left[\mathbf{p}_{e}(t), \mathbf{P}(t), \mathbf{e}(t+\delta t / 2),\right.\right. \\
& \mathbf{Q}(t+\delta t / 2)]+\widetilde{f}_{e_{\alpha}}\left[\mathbf{p}_{e}(t+\delta t), \mathbf{P}(t+\delta t),\right. \\
& \mathbf{e}(t+\delta t / 2), \mathbf{Q}(t+\delta t / 2)]\}-2 \delta t \lambda e_{\alpha}(t+\delta t / 2) .
\end{aligned}
$$

These equations also must be solved self-consistently since the forces $f_{j \alpha}$ and $\widetilde{f}_{e_{\alpha}}$ depend upon $\mathbf{p}_{e}$ through the vibrational eigenfunction. This is done using an analogous iterative approach as described for solving Eq. (A10) with two measures of convergence taken as $\Delta_{p_{e}}=\mid \mathbf{p}_{e, \text { input }}(t+\delta t)$ $-\left.\mathbf{p}_{e, \text { output }}(t+\delta t)\right|^{2} /\left|\mathbf{p}_{e, \text { output }}(t+\delta t)\right|^{2}$ and $\Delta_{P}=\mid \mathbf{P}_{\text {input }}(t+\delta t)$ $-\left.\mathbf{P}_{\text {output }}(t+\delta t)\right|^{2} /\left|\mathbf{P}_{\text {output }}(t+\delta t)\right|^{2}$. The method for determining $\lambda$ is given below.

Finally, update the coordinates from $t+\delta t / 2$ to $t+\delta t$

$$
\begin{aligned}
& Q_{j \alpha}(t+\delta t)=Q_{j \alpha}(t+\delta t / 2)+\frac{\delta t}{2} \frac{P_{j \alpha}(t+\delta t)}{m_{j}}, \\
& e_{\alpha}(t+\delta t)=e_{\alpha}(t+\delta t / 2)+\frac{\delta t}{2} B(t+\delta t / 2) p_{e_{\alpha}}(t+\delta t) .
\end{aligned}
$$

These equations are explicit.

We can use Eq. (A14) to determine the Lagrange multiplier $\lambda$ by requiring that $|\mathbf{e}(t+\delta t)|^{2}=1$. Defining the vector

$$
\begin{aligned}
\mathbf{e}_{0}(t+\delta t)= & \left.\mathbf{e}(t+\delta t)\right|_{\lambda=0}=\mathbf{e}(t+\delta t / 2)+\frac{\delta t}{2} B(t+\delta t / 2) \\
& \times\left[\mathbf{p}_{e}(t)+\delta t \widetilde{\mathbf{f}}_{e}(t+\delta t / 2)\right],
\end{aligned}
$$

we have

$$
\mathbf{e}(t+\delta t)=\mathbf{e}_{0}(t+\delta t)-\lambda \delta t^{2} B(t+\delta t / 2) \mathbf{e}(t+\delta t / 2) .
$$

It is not hard to show then that requiring that $\mathbf{e}(t+\delta t)$ is normalized leads to a quadratic equation in $\lambda$ with the solution 


$$
\lambda=\frac{D}{\delta t^{2} N_{1 / 2} B(t+\delta t / 2)}\left\{1-\left[1-\frac{N_{1 / 2}\left(N_{0}-1\right)}{D^{2}}\right]^{1 / 2}\right\},
$$

where $\quad D=\mathbf{e}_{0}(t+\delta t) \cdot \mathbf{e}(t+\delta t / 2), \quad N_{0}=\left|\mathbf{e}_{0}(t+\delta t)\right|^{2}, \quad$ and $N_{1 / 2}=|\mathbf{e}(t+\delta t / 2)|^{2}$. Note that the root of the quadratic equation with the negative sign is chosen so that when $N_{0}=1$, $\lambda=0$. This expression is used to calculate $\lambda$ in Eq. (A12); note that it only involves quantities at $t+\delta t / 2$ except for $\mathbf{p}_{e}(t+\delta t)$, which is obtained iteratively.

We note that for all the simulations presented here, the iterative solutions of both Eqs. (A10) and (A12) are completed in a single cycle. This may not be true for systems that exhibit stronger solute-solvent coupling.

${ }^{1}$ W. H. Thompson, Chem. Phys. Lett. 350, 113 (2001).

${ }^{2}$ R. Kubo, in Fluctuation, Relaxation and Resonance in Magnetic Systems, edited by D. ter Haar (Oliver and Boyd, London, 1962).

${ }^{3}$ A. Laubereau, Chem. Phys. Lett. 27, 600 (1974); S. F. Fischer and A. Laubereau, ibid. 35, 6 (1975); D. von der Linde, A. Laubereau, and W. Kaiser, Phys. Rev. Lett. 26, 954 (1971).

${ }^{4}$ R. M. Lynden-Bell, Mol. Phys. 33, 907 (1976).

${ }^{5}$ K. S. Schweizer and D. Chandler, J. Chem. Phys. 76, 2296 (1982).

${ }^{6}$ J. L. Skinner and D. Hsu, J. Phys. Chem. 90, 4931 (1986).

${ }^{7}$ J. Chesnoy and J. J. Weis, J. Chem. Phys. 84, 5378 (1986).

${ }^{8}$ S. J. Schvaneveldt and R. F. Loring, J. Chem. Phys. 102, 2326 (1995); R. B. Williams and R. F. Loring, ibid. 110, 10899 (1999).

${ }^{9}$ D. W. Oxtoby, D. Levesque, and J.-J. Weis, J. Chem. Phys. 68, 5528 (1978).

${ }^{10}$ D. W. Oxtoby, Adv. Chem. Phys. 40, 1 (1979); D. W. Oxtoby, in Vibrational Spectroscopy of Molecular Liquids and Solids, edited by S. Bratos and R. M. Pick (Plenum, New York, 1980); D. Levesque, J.-J. Weis, and D. W. Oxtoby, J. Chem. Phys. 79, 917 (1983).

${ }^{11}$ J. P. J. Michels and J. A. Schouten, Mol. Phys. 91, 253 (1997); M. E. Kooi, F. Smit, J. P. J. Michels, and J. A. Schouten, J. Chem. Phys. 112, 1395 (2000).

${ }^{12}$ N. Gayathri and B. Bagchi, J. Phys. Chem. A 103, 9579 (1999).

${ }^{13}$ K. F. Everitt and J. L. Skinner, J. Chem. Phys. 115, 8531 (2001).

${ }^{14}$ D. W. Oxtoby, Adv. Chem. Phys. 47, 487 (1981).

${ }^{15}$ J. C. Owrutsky, D. Raftery, and R. M. Hochstrasser, Annu. Rev. Phys. Chem. 45, 519 (1994).

${ }^{16}$ N. Halberstadt, S. Serna, O. Roncero, and K. C. Janda, J. Chem. Phys. 97, 341 (1992).

${ }^{17}$ R. Rey and J. T. Hynes, J. Chem. Phys. 108, 142 (1998); 104, 2356 (1996); R. M. Whitnell, K. R. Wilson, and J. T. Hynes, J. Phys. Chem. 94, 8625 (1990)

${ }^{18}$ B. J. Cherayil and M. D. Fayer, J. Chem. Phys. 107, 7642 (1997).

${ }^{19}$ G. Goodyear and S. C. Tucker, J. Chem. Phys. 110, 3643 (1999); S. C. Tucker, Chem. Rev. 99, 391 (1999).

${ }^{20}$ D. Secrest and B. R. Johnson, J. Chem. Phys. 45, 4556 (1966).

${ }^{21}$ A. A. Buchachenko, N. F. Stepanov, B. L. Grigorenko, and A. V. Nemukhin, J. Chem. Phys. 111, 2470 (1999).

${ }^{22}$ B. Faltermeier, R. Protz, M. Maier, and E. Werner, Chem. Phys. Lett. 74, 425 (1980); B. Faltermeier, R. Protz, and M. Maier, Chem. Phys. 62, 377 (1981).

${ }^{23}$ K. F. Everitt, S. A. Egorov, and J. L. Skinner, Chem. Phys. 235, 115 (1998); K. F. Everitt and J. L. Skinner, ibid. 110, 4467 (1999).

${ }^{24}$ S. A. Egorov and J. L. Skinner, Chem. Phys. Lett. 293, 469 (1998); S. A. Egorov, K. F. Everitt, and J. L. Skinner, J. Phys. Chem. A 103, 9494 (1999); J. L. Skinner and K. Park, J. Phys. Chem. B 105, 6716 (2001); K. F. Everitt, J. L. Skinner, and B. M. Ladanyi, J. Chem. Phys. 116, 179 (2002).
${ }^{25}$ J. S. Bader and B. J. Berne, J. Chem. Phys. 100, 8359 (1994).

${ }^{26}$ D. Rostkier-Edelstein, P. Graf, and A. Nitzan, J. Chem. Phys. 107, 10470 (1997).

${ }^{27}$ E. Rabani, S. A. Egorov, and B. J. Berne, J. Chem. Phys. 109, 6376 (1998); E. Rabani, S. A. Egorov, and B. J. Berne, J. Phys. Chem. A 103, 9539 (1999); S. A. Egorov, E. Rabani, and B. J. Berne, J. Phys. Chem. B 103, 10978 (1999).

${ }^{28}$ J. C. Tully, J. Chem. Phys. 93, 1061 (1990).

${ }^{29}$ J. C. Tully, in Modern Methods for Multidimensional Dynamics Computations in Chemistry, edited by D. L. Thompson (World Scientific, New Jersey, 1998).

${ }^{30}$ H.-D. Meyer and W. H. Miller, J. Chem. Phys. 70, 3214 (1979).

${ }^{31}$ F. Webster, E. T. Wang, P. J. Rossky, and R. A. Friesner, J. Chem. Phys. 100, 4835 (1994).

${ }^{32}$ O. V. Prezhdo and P. J. Rossky, J. Chem. Phys. 107, 825 (1997); K. F. Wong and P. J. Rossky, J. Phys. Chem. A 105, 2546 (2001).

${ }^{33}$ S. Nielsen, R. Kapral, and G. Ciccotti, J. Chem. Phys. 112, 6543 (2000).

${ }^{34}$ S. Hammes-Schiffer and J. C. Tully, J. Chem. Phys. 101, 4657 (1994).

${ }^{35}$ S. Hammes-Schiffer, J. Phys. Chem. A 102, 10443 (1998).

${ }^{36}$ A. Bastida, J. Zuñiga, A. Requena, I. Sola, N. Halberstadt, and J. A. Beswick, Chem. Phys. Lett. 280, 185 (1997); A. Bastida, J. Zuñiga, A. Requena, N. Halberstadt, and J. A. Beswick, J. Chem. Phys. 109, 6320 (1998); A. Bastida, B. Miguel, J. Zuñiga, A. Requena, N. Halberstadt, and K. C. Janda, ibid. 111, 4577 (1999).

${ }^{37}$ J. Ka and S. Shin, J. Phys. Chem. A 102, 3820 (1998).

${ }^{38}$ T. Terashima, M. Shiga, and S. Okazaki, J. Chem. Phys. 114, 5663 (2001).

${ }^{39}$ See, e.g., A. Messiah, Quantum Mechanics, Vol II (Wiley, New York, 1966).

${ }^{40}$ D. Borgis, G. Tarjus, and H. Azzouz, J. Phys. Chem. 96, 3188 (1992); D. Borgis, G. Tarjus, and H. Azzouz, J. Chem. Phys. 97, 1390 (1992).

${ }^{41}$ D. Laria, G. Ciccotti, M. Ferrario, and R. Kapral, J. Chem. Phys. 97, 378 (1992).

${ }^{42}$ S. P. Webb and S. Hammes-Schiffer, J. Chem. Phys. 113, 5214 (2000).

${ }^{43}$ M. F. Herman, J. Chem. Phys. 87, 4779 (1987); 87, 4794 (1987); M. F. Herman, Int. J. Quantum Chem. 70, 897 (1998).

${ }^{44}$ M. F. Herman, J. Chem. Phys. 109, 4726 (1998).

${ }^{45}$ R. B. Gerber, V. Buch, and M. A. Ratner, J. Chem. Phys. 77, 3022 (1982).

${ }^{46}$ B. J. Berne and D. Thirumalai, Annu. Rev. Phys. Chem. 37, 401 (1986).

${ }^{47}$ G. Wahnström, B. Carmeli, and H. Metiu, J. Chem. Phys. 88, 2478 (1988); K. Haug and H. Metiu, ibid. 97, 4781 (1992).

${ }^{48}$ R. Kosloff and A. D. Hammerich, Faraday Discuss. Chem. Soc. 91, 239 (1991).

${ }^{49}$ T. N. Truong, J. A. McCammon, D. J. Kouri, and D. K. Hoffman, J. Chem. Phys. 96, 8136 (1992).

${ }^{50}$ P. Nettesheim, F. A. Bornemann, B. Schmidt, and C. Schutte, Chem. Phys. Lett. 256, 581 (1996).

${ }^{51}$ D. Antoniou and S. D. Schwartz, J. Chem. Phys. 104, 3526 (1996).

${ }^{52} \mathrm{H}$. Goldstein, Classical Mechanics (Addison-Wesley, Reading, MA, 1980).

${ }^{53}$ R. P. Feynman, Phys. Rev. 56, 340 (1939).

${ }^{54}$ C. Lanczos, J. Res. Natl. Bur. Stand. 45, 255 (1950); Y. Saad, Numerical Methods for Large Eigenvalue Problems (Halstead, New York, 1992).

${ }^{55}$ J. Echave and D. C. Clary, Chem. Phys. Lett. 190, 225 (1992).

${ }^{56}$ H. Wei and T. Carrington, Jr., J. Chem. Phys. 97, 3029 (1992).

${ }^{57}$ D. T. Colbert and W. H. Miller, J. Chem. Phys. 96, 1982 (1992).

${ }^{58}$ A. S. Dickinson and P. R. Certain, J. Chem. Phys. 49, 4209 (1968); R. Meyer, ibid. 52, 2053 (1970).

${ }^{59}$ J. C. Light, I. P. Hamilton, and J. V. Lill, J. Chem. Phys. 82, 1400 (1985).

${ }^{60}$ M. F. Herman and B. J. Berne, J. Chem. Phys. 78, 4103 (1983).

${ }^{61}$ J. M. Sanz-Serna and M. P. Calvo, Numerical Hamiltonian Problems (Chapman \& Hall, New York, 1994).

${ }^{62}$ See also, J. B. Sturgeon and B. B. Laird, J. Chem. Phys. 112, 3474 (2000). 